PAPER

\title{
Does bilateral stimulation of the subthalamic nucleus aggravate apathy in Parkinson's disease?
}

\author{
V Czernecki, B Pillon, J L Houeto, M L Welter, V Mesnage, Y Agid, B Dubois
}

See Editorial Commentary, p 759

J Neurol Neurosurg Psychiatry 2005;76:775-779. doi: 10.1136/jnnp.2003.033258

See end of article for

authors' affiliations

......................

Correspondence to:

Virginie Czernecki,

INSERM U 610, Pavillon

Claude Bernard, Hôpital

de la Salpêtrière, 47

Boulevard de l'Hôpital,

75651 Paris cedex 13,

France; vczernecki@

hotmail.com

Received

27 November 2003

Revised version received

15 July 2004

Accepted 27 August 2004

\begin{abstract}
Objective: High frequency stimulation of the subthalamic nucleus (STN) dramatically decreases motor disability in patients with Parkinson"s disease (PD), but has been reported to aggravate apathy. The aim of this study was to analyse the effect of STN stimulation on motivation and reward sensitivity in a consecutive series of PD patients.

Methods: Apathy and reward sensitivity (Apathy Scale, Stimulus-Reward Learning, Reversal, Extinction, and Gambling tasks) were assessed in 18 PD patients treated by bilateral STN stimulation ("on" and "off" conditions) compared with 23 matched patients undergoing long term treatment with levodopa ("on" and "off" conditions).

Results: Apathy decreased under both STN stimulation and levodopa treatment, whereas explicit and implicit stimulus reward learning was unchanged.

Conclusions: Bilateral STN stimulation in PD patients does not necessarily have a negative effect on motivation and reward sensitivity and can even improve apathy provided patients have been appropriately selected for neurosurgery.
\end{abstract}

B ilateral high frequency stimulation of the subthalamic nucleus (STN) is an increasingly popular neurosurgical technique that decreases the severity of parkinsonian motor disability and levodopa induced motor complications by $60-80 \%$ and the required daily doses of levodopa by $40-$ $80 \%{ }^{1}$ It has been proposed that the improvement of motor symptoms results from inhibition of the hyperactivity of the STN, thereby restoring deficient thalamocortical activation of the supplementary motor area during movement. As the motor, associative, and limbic components of the corticalbasal ganglia-cortical loops pass through the STN it is unlikely that the restricted stimulation of such a small target selectively influences the motor component without also affecting the associative and limbic neuronal systems. ${ }^{2}$ The effects of stimulation of the STN on the associative component include an improvement of psychomotor speed and working memory together with an impairment of the ability to inhibit inappropriate responses. ${ }^{3-5}$ Stimulation of the STN also influences the cortico-subcortical limbic circuitry, ${ }^{6}$ as suggested by the appearance ${ }^{7-10}$ or improvement $^{11}{ }^{12}$ of various psychic disturbances.

Apathy, defined as a motivation in affect, cognition and behaviour, ${ }^{13}$ is a characteristic feature of Parkinson's disease $(\mathrm{PD})^{14}$ that decreases under levodopa treatment ${ }^{15}$ but remains unchanged or aggravated under bilateral STN stimulation. ${ }^{16-18}$ Whether this change in motivation results from a direct effect of STN stimulation or from the decrease in the daily dose of levodopa remains unclear. ${ }^{12}$ Nevertheless, the occurrence of apathy after neurosurgery may partially offset the benefit of this treatment. The aim of this study was to compare the effects of stimulation of the STN to those of levodopa treatment on apathy and reward sensitivity in PD patients.

\section{PATIENTS}

Eighteen consecutive patients treated by bilateral stimulation of the STN were recruited for the study. The neurosurgical procedure was performed as previously described. ${ }^{19}$ The electrodes were accurately implanted in the STN as shown by post surgery magnetic resonance imaging (MRI). Stimulation was effective because it resulted in a significant improvement in the motor score in the "off-levodopa" state and enabled the dose of levodopa to be significantly decreased (Table 1).

Before surgery, the motor disability score (UPDRS Part III $)^{20}$ was evaluated in the "off-levodopa" state, as defined by the Core Assessment Program for Surgical Interventional Therapy (CAPSIT). ${ }^{21}$ Ten months after surgery (mean \pm SEM: $10.0 \pm 0.9$ ) parkinsonian motor disability and performance on the experimental procedure were evaluated in the following conditions: 1) "off-stimulation" (after stimulation had been switched off for at least 1 hour) and "off-levodopa" (after a night without drug treatment)that is, deep brain stimulation (DBS) off, drugs off; 2) "onstimulation" (after stimulation had been switched on for at least 1 hour) and "off-levodopa" - that is, DBS on, drugs off.

Twenty three consecutive levodopa treated patients, most of them being candidates for neurosurgery, were matched to the stimulated patients for age, duration of disease, motor disability, and cognitive functions (Table 1) and tested "on" and "off" levodopa.

None of the patients was demented (score $>136$ on the Mattis Dementia Rating Scale) ${ }^{22}$ or depressed (score $<18$ on the Beck or the Montgomery and Asberg depression rating scales). ${ }^{23}{ }^{24} \mathrm{~A}$ "frontal score", ${ }^{25}$ modified to rate patients on a 50 point scale, $^{26}$ included the Modified Wisconsin Card Sorting Test, ${ }^{27}$ category and phonemic fluencies (animal names and words beginning with $M$ in 60 seconds), ${ }^{28}$ and graphic and motor series. ${ }^{29}$ Verbal learning was assessed with a procedure sensitive to the frontal strategic components of episodic memory. ${ }^{30}$ No significant difference was observed between stimulated and levodopa treated patients.

Abbreviations: DBS, deep brain stimulation; CAPSIT, Core Assessment Program for Surgical Interventional Therapy; MRI, magnetic resonance imaging; PD, Parkinson's disease; STN, subthalamic nucleus 
Table 1 Neurological and neuropsychological characteristics of parkinsonian patients treated by bilateral stimulation of the subthalamic nucleus or with levodopa

\begin{tabular}{|c|c|c|c|}
\hline & Stimulation & Levodopa & p Value \\
\hline Number of patients & 18 & 23 & \\
\hline Age at disease onset (years) & $45.5 \pm 1.2$ & $42.6 \pm 2.2$ & $>0.10$ \\
\hline Duration of disease (years) & $12.7 \pm 1.0$ & $14.9 \pm 1.2$ & $>0.10$ \\
\hline \multicolumn{4}{|c|}{ Parkinsonian motor disability (UPDRS-III) } \\
\hline \multicolumn{4}{|c|}{ Before neurosurgery } \\
\hline "off-levodopa" & $35.6 \pm 3.5$ & $38.7 \pm 2.8$ & $>0.10$ \\
\hline \multicolumn{4}{|l|}{ After neurosurgery } \\
\hline "on-stimulation-off-levodopa" & $10.1 \pm 2.4$ & & \\
\hline $\begin{array}{l}\text { "off-stimulation-off-levodopa" } \\
\text { Dose of levodopa (mg/day) }\end{array}$ & $33.0 \pm 3.1$ & & \\
\hline \multicolumn{4}{|l|}{ Dose of levodopa (mg/day) } \\
\hline Before neurosurgery & $843.3 \pm 77.6$ & $982.3 \pm 53.9$ & $>0.10$ \\
\hline & $133.3 \pm 58.7$ & \multicolumn{2}{|c|}{ Cognitive functions } \\
\hline Mattis Dementia Rating Scale & $141.0 \pm 0.3$ & $139.1 \pm 0.8$ & $>0.10$ \\
\hline Frontal score & $45.2 \pm 1.3$ & $46.5 \pm 0.8$ & $>0.10$ \\
\hline Verbal free recall & $28.2 \pm 1.5$ & $25.1 \pm 1.5$ & $>0.10$ \\
\hline Delayed free recall & $10.4 \pm 0.5$ & $10.1 \pm 0.6$ & $>0.10$ \\
\hline
\end{tabular}

To compare the effects of STN stimulation and levodopa on apathy and reward sensitivity, the patients were randomly assessed either in the "on" state and a day after in the "off" state or in the "off" state and a day after in the "on" state. They were assessed early in the morning before taking their usual drugs.

The Ethical Committee of the Salpêtrière Hospital approved the study and all subjects gave informed written consent.

\section{METHODS}

There were two assessments at a 24 hour interval for each patient. Each assessment lasted about 40 minutes allowing the patients to stay in a stable motor state. For each assessment, the tests were presented in the same order: Apathy Scale, Stimulus Reward Learning 1 and Reversal, Gambling task, Stimulus Reward Learning 2, and Extinction.

\section{Apathy scale}

Fourteen questions-for example, "Do you have plans and goals for the future?"-were read by the examiner. For each question, the subject was given four possible answers: "not at all", "slightly", "some", or "a lot". Scores ranged from 0 to 42 , with higher scores indicating more apathy. A score of 14 was used as a pathological cut off level. Subjects were asked to answer according to how they felt at the time of the examination. This scale has been shown to be reliable for the evaluation of apathy in PD. ${ }^{31}$

\section{Stimulus Reward Learning, Reversal, and Extinction}

The tasks were adapted from Rolls et al. ${ }^{32}$ In Stimulus Reward Learning 1, the subject first learned to touch one of two highly discriminable coloured fractal images that appeared randomly on a video monitor equipped with a touch screen. Different patterns were used for the first and second assessment. The subject gained one point for touching the correct pattern or not touching the incorrect one, and lost one point for not touching the correct pattern or touching the incorrect one. If the pattern was touched, it was immediately replaced by a message telling the subject whether a point had been gained or lost. If the pattern was not touched it disappeared after 7 seconds and was replaced by a message telling the subject whether a point had been gained or lost. A pleasant rising tone also emphasised correct responses, whereas incorrect responses were signalled by a short unpleasant tone. A running total of obtained points was displayed on the screen. The subjects were asked to try to gain as many points as possible. They advanced to each new trial at their own pace, by pressing the space bar on a keyboard, until a criterion of nine correct responses out of ten trials had been reached. The score consisted of the number of trials needed to attain the criterion.

Once the Stimulus Reward Learning criterion had been reached, the Reversal task automatically occurred without warning-the relationship between the patterns and the rewarding or punishing consequences being reversed. Testing continued for 30 trials and further reversals occurred whenever the criterion of nine successive correct responses was reached again. The scores consisted of the number of reversals in 30 trials, the number of trials and the number of errors for the first reversal, the last error trial for the first reversal, and the total number of commission errors (previously correct stimuli touched) and omission errors (previously incorrect stimuli not touched). Reversal has been shown to reflect the ability to shift a mental set on the basis of affective cues (affective shifting) in contrast to tests such as the Wisconsin Card Sorting Test that instead evaluate the ability to shift on the basis of a cognitive process (attentional shifting). ${ }^{33}$

Stimulus Reward Learning 2, before Extinction, consisted of the same procedure as before Reversal, but with different patterns. After the Stimulus Reward Learning criterion had been reached, the Extinction task automatically took place. In this condition points were won each time the subject refrained from touching one pattern and were lost by touching it. The scores were the number of trials needed, the last error trial, the total number of perseveration errors (previously correct stimuli touched), and attribution errors (previously incorrect stimuli touched). The Extinction task provides a means of estimating the control of impulsivity. Interestingly, deficits in reversal and extinction have been shown to be specifically related to impaired reward sensitivity and to dysfunction of the orbitofrontal cortex, but not of the dorsolateral prefrontal cortex, from evidence drawn from experimental lesions in animals and from focal lesions in humans. ${ }^{3435}$

\section{The Gambling task}

Bechara et al provided the computerised version of the task. ${ }^{36}$ The subject sees on the screen four decks of cards labelled A, B, C, and D, each of them being programmed to have 60 cards. Using a mouse, he or she can click on a card from any of the four decks. Every time the subject picks a card, a 
Table 2 Effects of bilateral stimulation of the subthalamic nucleus and levodopa treatment on apathy and reward sensitivity

\begin{tabular}{|c|c|c|c|c|c|}
\hline & Stimulation & Levodopa & Group ( $p$ value) & Treatment ( $p$ value) & Inter ( $p$ value) \\
\hline \multicolumn{6}{|c|}{ 1. Apathy Scale (total score) } \\
\hline "on" treatment & $11.2 \pm 0.9$ & $11.0 \pm 1.5$ & 0.91 & $<0.0001$ & 0.55 \\
\hline "off" treatment & $13.4 \pm 1.2$ & $14.0 \pm 1.5$ & & & \\
\hline \multicolumn{6}{|c|}{ 2. Stimulus Reward Association } \\
\hline \multicolumn{6}{|c|}{ Learning (number of trials) } \\
\hline "on" treatment & $19.2 \pm 3.9$ & $22.6 \pm 5.6$ & 0.37 & 0.30 & 0.81 \\
\hline "off" treatment & $23.1 \pm 4.6$ & $28.8 \pm 4.8$ & & & \\
\hline \multicolumn{6}{|c|}{ Reversal (number in 30 trials) } \\
\hline "on" treatment & $1.6 \pm 0.2$ & $1.3 \pm 0.2$ & 0.48 & 0.28 & 0.43 \\
\hline "off" treatment & $1.3 \pm 0.2$ & $1.3 \pm 0.2$ & & & \\
\hline \multicolumn{6}{|l|}{ Extinction (last error) } \\
\hline "on" treatment & $8.1 \pm 1.1$ & $14.2 \pm 2.5$ & 0.13 & 0.99 & 0.13 \\
\hline \multicolumn{6}{|c|}{ 3. Gambling Task (advantageous minus disadvantageous choices) } \\
\hline "on" treatment & $25.4 \pm 10.2$ & $13.4 \pm 6.9$ & 0.39 & 0.65 & 0.56 \\
\hline "off" treatment & $19.4 \pm 9.6$ & $14.2 \pm 6.4$ & & & \\
\hline
\end{tabular}

Values expressed as mean \pm SEM. The ANOVA results are shown as probability levels for the group effect (patients with stimulation versus patients with levodopa), the treatment effect (on treatment/off treatment), and the interaction between these two factors (Inter).

message is displayed on the screen indicating the amount of money he or she has won or lost. A green bar on the top of the screen also changes according to the amount of money won or lost. The subject is asked to win as much money as possible, and, if he or she cannot, to avoid losing money as much as possible. The experiment shuts off automatically when 100 cards have been selected. The subject must progressively discover that decks A and B are disadvantageous (big gains but bigger losses), whereas decks C and D are advantageous (small gains but even smaller losses). The scores consisted of the number of advantageous choices $(\mathrm{C}+\mathrm{D})$ minus disadvantageous choices $(\mathrm{A}+\mathrm{B})$ for each of the five blocks of 20 cards and for the total of the 100 cards. Deficits on this task have been shown to be related to lesions or dysfunction of the orbitofrontal cortex and to be independent of working memory deficits, which are related to lesions of the prefrontal dorsolateral cortex. ${ }^{37}$

\section{Data analysis}

The same analyses were performed for all variables: 1) analysis of the effects of stimulation using ANOVA with repeated measures ("on-stimulation" state versus "offstimulation" state); 2) comparison of stimulated patients who improved or not improved under stimulation and of apathetic and non-apathetic patients between groups using ANOVA; 3) comparison of the effects of stimulation with those of levodopa, using ANOVA with repeated measures, with the two groups (stimulated patients versus levodopa treated patients) as a between factor and condition of treatment ("on" treatment [with stimulation or levodopa] versus "off" treatment [without stimulation or levodopa]) as a within factor. To evaluate apathy score changes under stimulation among individual patients each test score was transformed to a standard z score, using total sample baseline means and standard deviation (SD). The clinical criterion of more than 1.0 SD above or below the mean was used to tally impro-

vement or deterioration under stimulation. Correlations were searched for between the severity of apathy and all the cognitive or clinical characteristics of the stimulated patients.

\section{RESULTS}

\section{Influence of STN stimulation on apathy and reward sensitivity}

The apathy score significantly improved under STN stimulation $(\mathrm{F}(1,16)=8.5 ; \mathrm{p}=0.01)$, but the reward sensitivity scores did not change (Table 2). As the state of stimulation and the order of assessment could interact with the performance on Stimulus Reward Learning, Reversal and Extinction, and the Gambling task, we reanalysed these variables, comparing the two subgroups on the first assessment only. No difference in these variables was noted between the "on-stimulation" state and the "off-stimulation" state. Overall, these results show an improvement of apathy under stimulation, but no change of reward sensitivity.

Individual results showed a significant improvement of apathy under stimulation in nine patients, no significant change in eight patients, and a significant aggravation in only one patient. The nine patients who significantly improved differed from the other patients in only two ways: a shorter duration of the disease $(10.4 \pm 1.4$ versus $15.0 \pm 1.0$ years; $\mathrm{F}(1,16)=6.5 ; \mathrm{p}=0.02)$ and a lower UPDRS score without stimulation and without treatment $(26.3 \pm 4.3$ versus $39.7 \pm 3.4 ; \mathrm{F}(1,16)=5.9 ; \mathrm{p}=0.03)$. They did not differ from the other patients for the doses of levodopa or of dopaminergic agonists converted in Levodopa Equivalent Doses (Table 3; $6 \mathrm{mg}$ of ropinirol $=100 \mathrm{mg}$ of levodopa; $1 \mathrm{mg}$ of pergolid $=100 \mathrm{mg}$ of levodopa; $10 \mathrm{mg}$ of bromocriptine $=100 \mathrm{mg}$ of levodopa). ${ }^{38}{ }^{39}$ In the group with improvement of apathy under stimulation, eight patients received dopaminergic agonists (ropinirol for two patients with a mean daily dose of $21.0 \pm 9.0 \mathrm{mg}$; pergolid for five patients with a mean daily dose of $1.9 \pm 0.6 \mathrm{mg}$; bromocriptin for one patient with a mean daily dose of $40 \pm 0.0 \mathrm{mg}$ ). In the group without improvement of apathy under stimulation, six patients received dopaminergic agonists (ropinirol for three patients with a mean daily dose of $8.3 \pm 2.3 \mathrm{mg}$; pergolid for two patients with a mean daily dose of $2.2 \pm 0.7 \mathrm{mg}$; bromocriptin for one patient with a mean daily dose of $40 \pm 0.0 \mathrm{mg}$ ). None of the stimulated patients received other drugs.

\section{Relation between severity of apathy and clinical and experimental variables in stimulated patients}

At baseline, six stimulated patients (33\%) had an apathy score higher than 14-a score considered as pathological. ${ }^{31}$ They were significantly more impaired $(p<0.05)$ than the other stimulated patients on all variables of Reversal. This was not the case for Stimulus Reward Learning or the Gambling task.

A matrix of correlation showed significant correlations $(p<0.05)$ between the severity of apathy and the frontal 
Table 3 Treatment of patients whose apathy improved or not improved under stimulation

\begin{tabular}{llll}
\hline & Improved & Not improved & p Value \\
\hline Number of patients & 9 & 9 & \\
Levodopa (mg/day) & $161.1 \pm 86.5$ & $105.6 \pm 83.5$ & $>0.10$ \\
Agonists (LED mg/day) & $230.5 \pm 57.2$ & $140.7 \pm 47.3$ & $>0.10$ \\
Total treatment (LED mg/day) & $391.7 \pm 133.1$ & $246.2 \pm 81.3$ & $>0.10$ \\
\hline
\end{tabular}

Values expressed as mean \pm SEM; LED, mean daily dose of levodopa equivalent. ${ }^{38}{ }^{39}$

Total treatment: sum of levodopa and dopaminergic agonists converted in LED.

score, the number of perseverations on the Wisconsin Card Sorting test, and the total recall score of the Grober and Buschke test. In contrast, no correlation was found between the severity of apathy and severity of depression, age at disease onset, duration of the disease, UPDRS scores with or without stimulation, or levodopa dose.

\section{Comparison of the effect of stimulation and that of levodopa}

There was no group effect and no interaction between group and condition (Table 2). The effect of treatment was limited to the apathy scale $(F(1,37)=18.7 ; p<0.0001)$. Overall, therefore, the results were similar for the two groups of patients, with an improvement of apathy both under stimulation and levodopa treatment, but no change in reward sensitivity; however, it must be highlighted that the group with levodopa was tested after withdrawal of $982 \mathrm{mg}$ of levodopa (plus dopaminergic agonists) whereas the group with stimulation was tested after withdrawal of $133 \mathrm{mg}$ of levodopa (plus dopaminergic agonists). Unfortunately the doses of dopaminergic agonists or other drugs were not collected for the group with levodopa.

\section{DISCUSSION}

At baseline (without treatment), 33\% of the patients treated by STN stimulation and 39\% of the patients treated by levodopa could be considered as apathetic (apathy score $>14)$. ${ }^{31}$ These results suggest, therefore, that apathy is not an inevitable consequence of neurosurgery. In addition, apathy improved under stimulation in patients with a moderate disease duration (about 10 years) and moderate disease severity (UPDRS III score "off stimulation" and "off levodopa" of about 26). The improvement of the apathy score was of the same magnitude whether patients were treated by STN stimulation or with levodopa (Table 2). This result is in contradiction with recent studies that showed an aggravation of apathy under STN stimulation. ${ }^{10}{ }^{17}$ In the latter studies, however, the patients had severe personality disorders before surgery ${ }^{10}$ or were older. ${ }^{17}$ We can conclude that apathy is improved by both STN stimulation and levodopa treatment provided strict inclusion criteria for neurosurgery are applied.

Does stimulation of the STN mimic the effects of levodopa treatment on motivation as it does in the cognitive and in the motor domains? This cannot be confirmed because long term effects of dopaminergic agonists or other drugs, likely more important in the levodopa group, could be a possible confounding issue. Furthermore, it has been shown that a suprathreshold dose of levodopa improved more apathy than STN stimulation. ${ }^{12}$ The doses of dopaminergic agonists did not differ, however, between patients whose apathy improved or not improved under STN stimulation and none of the stimulated patients received other drugs. The improvement of apathy might result from motor improvement. Recent studies, however, have shown that affective changes under STN stimulation were unrelated to motor improvement. ${ }^{78}$ This suggests that STN stimulation interacts with the functioning of neural pathways involved not only in motor and cognitive control but also in affective states, particularly the "anterior cingulate" striato-thalamo-cortical circuit, which has been shown to be related to apathy. ${ }^{40}$ The finding that STN stimulation increased activation of the anterior cingulate cortex during a response conflict task in a positron emission tomography study provides direct evidence of STN modulating non-motor basal ganglia-thalamo-cortical circuitry. ${ }^{41}$

Stimulation of the STN had no influence on Stimulus Reward Learning, Reversal, Extinction, or the Gambling Task. We cannot rule out the possibility that this was related to the short duration of withdrawal of the stimulation. The same pattern of response (improvement of apathy and lack of influence on Stimulus Reward Learning, Reversal, and Extinction) has, however, been observed with levodopa treatment although treatment was withdrawn for about 12 hours. This suggests: 1) that motivation and explicit and implicit reward associative learning are controlled by different striatofrontal circuits; and 2) that these neuronal circuits are differentially impaired in PD or differentially sensitive to treatment. Apathy would be more related to a dysfunction of the "cingulate" loop, ${ }^{40}$ whereas reward sensitivity would be more associated with a dysfunction of the "orbitofrontal" loop. ${ }^{36}$ Alternatively, experimental studies in monkeys have shown that physiological mechanisms of reward sensitivity imply phasic firing of dopaminergic neurons. ${ }^{42}$ Our data might suggest that the phasic response implicated in reward sensitivity is not compensated for by levodopa or by stimulation, in contrast to the tonic response required by motivation.

In conclusion, bilateral stimulation of the STN in PD patients does not necessarily have a negative effect on motivation or reward sensitivity, and can even improve apathy, possibly by activation of a cortico-subcortico-cortical associativo-limbic neuronal loop passing through the cingulate area, provided patients have been appropriately selected for surgery. ${ }^{1}$ Further studies are required, however, using the same patients as their own controls preoperatively and postoperatively, using a more global scale to assess the patients and carers impression of how they feel on and off stimulation, and searching for the possible influence of the exact electrodes location or dosage of levodopa or other drugs modulating dopaminergic or non-dopaminergic transmission.

\section{ACKNOWLEDGEMENTS}

INSERM (Institut National de la Santé et de la Recherche Médicale) and Assistance Publique supported the study. We thank A Bechara for providing us with the computerised version of his Gambling task, A M Bonnet and the nurses of the Fédération de Neurologie and Centre d'Investigation Clinique for their contribution. We are also grateful to Leon Tremblay and Mathias Pessiglione for helpful comments, Magali Volteau for statistical advice, and Nikki Horne for revising the English. 


\section{Authors' affiliations}

V Czernecki, B Pillon, B Dubois, INSERM U 610, Pavillon Claude Bernard, Hôpital de la Salpêtrière, 47 Boulevard de l'Hôpital, 75651

Paris cedex 13, France

B Pillon, J L Hoveto, M L Welter, V Mesnage, Y Agid, Centre d'Investigation Clinique, Fédération de Neurologie and INSERM U 289 Hôpital de la Salpêtrière, 47 Boulevard de l'Hôpital, 75651 Paris cedex 13, France

Competing interests: none declared

\section{REFERENCES}

1 Welter ML, Houeto JL, Tezenas du Moncel S, et al. Clinical predictive factors of subthalamic stimulation in Parkinson's disease. Brain 2002;125:575-83.

2 Baron MS, Wichmann T, Ma D, et al. Effects of transient focal inactivation of the basal ganglia in parkinsonian primates. J Neurosci 2002;22:592-9.

3 Ardouin C, Pillon B, Peiffer E, et al. Bilateral subthalamic or pallidal stimulation for Parkinson's disease affects neither memory nor executive functions: a consecutive series of 62 patients. Ann Neurol 1999:46:217-23.

4 Pillon B, Ardouin C, Damier Ph, et al. Neuropsychological changes between "off" and "on" STN or GPi stimulation in Parkinson's disease. Neurology 2000:55:411-18.

5 Jahanshahi M, Ardouin C, Brown RG, et al. The impact of deep brain stimulation on executive function in Parkinson's disease. Brain 2000;123:1142-54.

6 Alexander GE, Crutcher MD, Delong MR. Basal ganglia-thalamocortical circuits: parallel substrates for motor, oculomotor, "prefrontal" and "limbic" functions. Prog Brain Res 1990;85:119-46.

7 Beijani BP, Damier P, Arnulf I, et al. Transient acute depression induced by high-frequency deep-brain stimulation. New Eng J Med 1999;340:1476-80

8 Krack P, Kumar R, Ardouin C. Mirthful laughter induced by subthalamic nucleus stimulation. Mov Disord 2001;16:867-75.

9 Kulisevsky J, Berthier ML, Gironell A, et al. Secondary mania following subthalamic nucleus deep brain stimulation for the treatment of Parkinson's disease. Neurology 2001;56(suppl 43):A49.

10 Hoveto JL, Mesnage V, Mallet L, et al. Behavioral disorders, Parkinson's disease, and subthalamic stimulation. J Neurol Neurosurg Psychiatry 2002;72:701-7.

11 Mallet L, Mesnage V, Houeto JL, et al. Compulsions, Parkinson's disease and stimulation. Lancet 2002;360:1302-4

12 Funkiewiez A, Ardouin C, Krack P, et al. Acute psychotropic effects of bilateral subthalamic nucleus stimulation and levodopa in Parkinson's disease. Mov Disord 2003; 18:524-30.

13 Marin RS. Apathy: concept, syndrom, neural mechanisms and treatment. Semin Clin Neuropsychiatry 1996;1:304-14.

14 Pluck GC, Brown RG. Apathy in Parkinson's disease. J Neurol Neurosurg Psychiatry 2002;73:636-42.

15 Czernecki V, Pillon B, Houeto Jl, et al. Motivation, reward and Parkinson's disease: influence of dopatherapy. Neuropsychologia 2002;40:2257-67.

16 Trépanier LL, Kumar R, Lozano AM, et al. Neuropsychological outcome of GPi pallidotomy and GPi or STN deep brain stimulation in Parkinson's disease. Brain Cogn 2000;42:324-47.

17 Saint-Cyr JA, Trépanier LL, Kumar R, et al. Neuropsychological consequences of chronic bilateral stimulation of the subthalamic nucleus in Parkinson's disease. Brain 2000;123:2091-108.

18 Pillon B. Neuropsychological assessment for management of patients with deep brain stimulation. Mov Disord 2002;17:S116-22.

19 Beijani BP, Dormont D, Pidoux B, et al. Bilateral subthalamic stimulation for Parkinson's disease by using three-dimensional stereotactic magnetic resonance imaging and electrophysiological guidance. J Neurosurg 2000;92:615-25.
20 Fahn S, Elton RL, UPDRS Development Committee. Unified Parkinson's disease rating scale. In: Fahn S, Marsden CD, Calne D, et al, eds. Recent Developments in Parkinson's Disease. Florham Park, NY: MacMillan Healthcare Information, 1987;2:153-63;293-304.

21 Defer GL, Widner H, Marie RM, et al. Core assessment program for surgical interventional therapies in Parkinson's disease(CAPSIT-PD). Mov Dis 1999;14:572-84

22 Mattis S. Dementia Rating Scale. Odessa, FL: Psychological Assessment Resources Inc, 1988.

23 Beck AT. Beck depression inventory. San Antonio, TX: The Psychological Corporation, 1987.

24 Montgomery SA, Asberg MA. A new depression scale designed to be sensitive to change. Br J Psychiatry 1979;134:382-9.

25 Pillon B, Gouider-Khouja N, Deweer B, et al. The neuropsychological pattern of striatonigral degeneration: comparison with Parkinson's disease and progressive supranuclear palsy. I Neurol Neurosurg Psychiatry 1995; $58: 174-9$

26 Limousin P, Krack P, Pollak P, et al. Electrical stimulation of the subthalamic nucleus in advanced Parkinson's disease. N Eng J Med 1988;339:1105-11

27 Nelson HE. A modified Card Sorting Test sensitive to frontal lobe defect. Cortex 1976;12:313-24.

28 Benton AL. Differential behavioral effects in frontal lobe disease. Neuropsychologia 1968;6:53-60.

29 Luria AR. Higher Cortical Functions in Man. New York, NY: Basic Books Inc Publishers, 1966

30 Grober E, Buschke H. Genuine memory deficits in dementia. Dev Neuropsychol 1987:3:13-36.

31 Starkstein SE, Mayberg HS, Preziosi TJ, et al. Reliability, validity and clinical correlates of apathy in Parkinson's disease. I Neuropsychiatry Clin Neurosci 1992;4:134-9

32 Rolls ET, Hornak J, Wade D, et al. Emotion-related learning in patients with social and emotional changes associated with frontal lobe damage. J Neurol Neurosurg Psychiatry 1994;57:1518-24.

33 Swainson R, Rogers RD, Sahakian BJ, et al. Probabilistic learning and reversal deficits in patients with Parkinson's disease or frontal or temporal lobe lesions: possible effects of dopaminergic medication. Neuropsychologia 2000;38:596-612.

34 Dias R, Robbins TW, Roberts AC. Dissociation in prefrontal cortex of affective and attentional shifts. Nature 1996;380:69-72.

35 Rolls ET. The Brain and Emotion. Oxford: Oxford University Press, 1999.

36 Bechara A, Damasio H, Damasio AR. Emotion, decision making and the prefrontal cortex. Cereb Cortex 2000;10:295-307.

37 Bechara A, Damasio H, Tranel D, et al. Dissociation of working memory from decision making within the human prefrontal cortex. J Neurosci 1998;18:428-37.

38 Goetz CG, Blasucci L, Stebbins GT. Switching dopamine agonists in advanced Parkinson's disease: is rapid titration preferable to slow? Neurology 1999:52:1227-9.

39 Gimenez-Roldan S, Esteban EM, Mateo D. Swithching from bromocriptine to ropinirole in patients with advanced Parkinson's disease: open label pilot responses to three different dose-ratios. Clin Neuropharmacol 2001;24:346-51.

40 Benoit M, Koulibaly PM, Migneco O, et al. Brain perfusion in Alzheimer's disease with and without apathy: a SPECT study with statistical parametric mapping analysis. Psychiatry Res Neuroimaging 2002;114:103-11.

41 Schroder U, Kuehler A, Haslinger B, et al. Subthalamic nucleus stimulation affects striato-anterior cingulate cortex circuit in a response conflict task: a PET study. Brain 2002;125:1995-2004.

42 Schultz W, Dayan P, Read Montague P. A neural substrate of prediction and reward. Science 1997:275:1593-9. 